\title{
A Randomized Trial of rTMS Targeted with MRI Based Neuro-Navigation in Treatment-Resistant Depression
}

\author{
Paul B Fitzgerald*,', Kate Hoy', Susan McQueen', Jerome J Maller', Sally Herring', Rebecca Segrave', \\ Michael Bailey², Greg Been', Jayashri Kulkarni' and Zafiris J Daskalakis ${ }^{3}$ \\ 'Alfred Psychiatry Research Centre, The Alfred and Monash University School of Psychology, Psychiatry and Psychological Medicine, Commercial \\ Rd Melbourne, Victoria, Australia; ${ }^{2}$ Monash University Department of Epidemiology and Preventive Medicine, The Alfred Hospital, Commercial Rd \\ Melbourne, Victoria, Australia; ${ }^{3}$ Centre for Addiction and Mental Health, University of Toronto, Toronto, Ontario, Canada
}

\begin{abstract}
The aim of this study is to investigate whether repetitive transcranial magnetic stimulation (rTMS) targeted to a specific site in the dorsolateral prefrontal cortex (DLPFC), with a neuro-navigational method based on structural MRI, would be more effective than rTMS applied using the standard localization technique. Fifty-one patients with treatment-resistant depression were randomized to receive a 3-week course (with a potential I-week extension) of high-frequency $(10 \mathrm{~Hz})$ left-sided rTMS. Thirty trains ( $5 \mathrm{~s}$ duration) were applied daily 5 days per week at $100 \%$ of the resting motor threshold. Treatment was targeted with either the standard $5 \mathrm{~cm}$ technique $(n=27)$ or using a neuro-navigational approach $(n=24)$. This involved localizing the scalp location that corresponds to a specific site at the junction of Brodmann areas 46 and 9 in the DLPFC based on each individual subject's MRI scan. There was an overall significant reduction in the Montgomery-Asberg Depression Rating Scale scores over the course of the trial, and a better outcome in the targeted group compared with the standard localization group at 4 weeks $(p=0.02)$. Significant differences were also found on secondary outcome variables. The use of neuro-navigational methods to target a specific DLPFC site appears to enhance response to rTMS treatment in depression. Further research is required to confirm this in larger samples, or to establish whether an alternate method based on surface anatomy, including measurement from motor cortex, can be substituted for the standard $5 \mathrm{~cm}$ method.

Neuropsychopharmacology (2009) 34, 1255-1262; doi: I0.1038/npp.2008.233; published online I4 January 2009
\end{abstract}

Keywords: repetitive transcranial magnetic stimulation; depression; prefrontal cortex; response; remission; antidepressant

\section{INTRODUCTION}

Major depressive disorder (MDD) is a substantive clinical problem complicated by the failure of about $30 \%$ of the patients to respond to standard medication treatments (Fava, 2003). Repetitive transcranial magnetic stimulation (rTMS), applied to the dorsolateral prefrontal cortex (DLPFC), has been investigated actively as an alternative intervention for patients with treatment-resistant depression (TRD) (eg George et al, 1995, 2000; Fitzgerald et al, 2003, 2006a). Most trials have suggested that the active rTMS treatment has greater antidepressant efficacy than sham stimulation (Fitzgerald, 2006), including a large international multi-site trial (O'Reardon et al, 2007). Despite this evidence, however, concerns about the efficacy and clinical relevance of these results continue to be

\footnotetext{
* Correspondence: Professor PB Fitzgerald, Alfred Psychiatry Research Centre, First Floor, Old Baker Building, The Alfred, Commercial Rd Melbourne, Victoria 3004, Australia, Tel: +61 39076 6552, Fax: +61 39076 6588, E-mail: paul.fitzgerald@med.monash.edu.au

Received 8 August 2008; revised 14 December 2008; accepted 16 December 2008
}

expressed, as many studies have reported limited effect sizes or response rates (Loo and Mitchell, 2005).

It is possible that the marginal response rates reported in some studies relate to the way in which the rTMS treatment is targeted over the DLPFC. Almost all studies to date have followed a standard procedure based on the earliest TMS investigations (George et al, 1995; Pascual-Leone et al, 1996). This involves the localization of the motor cortical site for optimal stimulation of a hand muscle, usually the abductor pollicis brevis, and then measurement $5 \mathrm{~cm}$ anteriorly along the scalp surface (' $5 \mathrm{~cm}$ method'). However, when the fidelity of this method was evaluated, it localized stimulation to DLPFC (defined as cortical landmarks corresponding to Brodmann area, BA, 9) in only 7 of 22 subjects (Herwig et al, 2001a). An even lower success rate would have been likely if a more modern definition of DLPFC was used based on multiple brains such as that published by Rajkowska et al (Rajkowska and GoldmanRakic, 1995). This analysis produced a considerably more circumscribed definition of areas 9 and 46. This suggests that the traditional methods for targeting the DLPFC with rTMS may be suboptimal, potentially explaining the modest therapeutic effects of rTMS. 
Neuro-navigational methods which can be used to improve targeting of rTMS, have recently been developed (Herwig et al, 2001b; Krings et al, 2001; Schonfeldt-Lecuona et al, 2005). These generally involve localization of the scalp position that corresponds to a brain site identified on an MRI scan from an individual subject. To date, only one published clinical trial has used neuro-navigational methods to target rTMS in TRD; but this study did not target DLPFC based on anatomy (Herwig et al, 2003). Therefore, we conducted a randomized double blind trial comparing left prefrontal rTMS localized either using the $5 \mathrm{~cm}$ method or a navigational technique. We hypothesized that the targeted rTMS would result in a greater therapeutic efficacy in MDD compared with the ' $5 \mathrm{~cm}$ ' method. We utilized an 'offline' navigational technique to localize the site of DLPFC in the experimental group and compared this with the $5 \mathrm{~cm}$ localization method.

\section{METHODS}

\section{Design}

The study involved a two-arm double blind randomized controlled trial $(n=51)$ conducted at a single site. Fifty-one subjects with TRD were randomized to the rTMS treatment provided with a standard localization procedure $(n=24)$ or a neuro-navigation-aided localization $(n=27)$, and were observed at baseline, and post-baseline weeks 3 and 4 . The localization was performed by a rater not involved in the treatment or assessments such that the patients, raters, and the clinicians administering rTMS were all blind to the treatment type. The patients were randomized sequentially using a single computer-generated random number sequence (no stratification). Coordinates were provided to the treater immediately prior to the first treatment session. The treatment duration was 15 daily sessions with a 5-day extension period for patients who achieved a $>25 \%$ reduction on the Montgomery-Asberg Depression Rating Scale (MADRS) (Montgomery and Asberg, 1979). The rationale for this was the assumption that patients who had not improved within 3 weeks would be unlikely to benefit from the additional period of treatment.

\section{Subjects}

Fifty-one outpatients were recruited at the Alfred Psychiatry Research Centre from the Alfred Department of Psychiatry and by referral from a number of private psychiatrists between December 2005 and April 2007. The diagnosis was made using the MINI (Sheehan et al, 1998) by a study psychiatrist. The inclusion criteria for the study included: age 18-70 years, a diagnosis of MDD (not bipolar disorder), and the presence of moderate to severe depression $(>20$ on the MADRS (Montgomery and Asberg, 1979)) without psychosis. In addition, all the patients were required to have failed to respond to a minimum of two courses of antidepressant medications for at least 6 weeks in the current episode (Stage II, Thase and Rush Definition (Thase and Rush, 1997); mean number of courses $=5.7 \pm 3.9$ ), as determined by their primary treating clinician and patient judgment of medication effectiveness. The exclusion criteria included a diagnosis of a significant active medical illness, any history of epilepsy or other neurological illness, or any contra-indication to MRI scanning. The patients were not allowed to change their medication during the trial or in the prior 4 weeks. Written informed consent was obtained from all the patients on a form approved by the Alfred Hospital/ Bayside Health Human Research Ethics committee. The study sample size was determined prior to the trial to provide sufficient power $(0.81)$ to find a 5.5 point difference between the groups on end-study MADRS scores $(\alpha<0.05$, $S D=7$ ), based on end-study scores for the full sample assuming a last observation carried forward analysis for dropouts. We believed that this was a clinically relevant end-point difference. The sample size calculation was an approximation of the required sample size only, as the analysis utilized more complex statistical methods than that used for the sample size calculation.

\section{Clinical Assessment}

The primary outcome measure for the study was scores on the MADRS (Montgomery and Asberg, 1979). Assessment was also completed with the Beck Depression Inventory (second version) (Beck et al, 1961), the Brief Psychiatric Rating Scale (Overall and Gorham, 1962), the CORE rating of psychomotor disturbance (Hickie et al, 1990), the Global Assessment of Functioning (GAF) (American Psychiatric Association, 1994), and the Clinical Global Improvements Scale (Guy, 1976).

A brief cognitive assessment was made at baseline and treatment end using the following tests (alterative versions used for repeat testing): the Hopkins Verbal Learning Test (Arnold et al, 1995), Controlled Oral Word Association Test (Benton, 1996), Digit Span (forwards and backwards) (Wechsler, 1997), and the Brief Visuospatial Memory Test-Revised (Benedict et al, 1996). Ratings were conducted by clinical-trained raters who were required to maintain adequate inter-rater reliability $\left(r^{2}>0.9\right)$.

\section{TMS Treatment}

rTMS was provided using a Medtronic Magpro30 magnetic stimulator (Medtronic Inc, MI, USA) using $70 \mathrm{~mm}$ figure-of8 coils held in place with a custom-made stand. The coil was held tangential to the scalp with the handle pointing back and away from the midline at $45^{\circ}$. The resting motor threshold was measured using standard visual methods (Pridmore et al, 1998). For all subjects, the rTMS treatment was applied at $10 \mathrm{~Hz}$ with an intensity of $100 \%$ of resting motor threshold. Thirty trains of $5 \mathrm{~s}$ duration with $25 \mathrm{~s}$ between trains were applied (1500 pulses per day, 30000 over 4 weeks).

\section{Stimulation Localization}

All the patients underwent a MRI scan (124 slice T1weighted sequence on a $1.5 \mathrm{~T}$ GE Signa scanner with six fiducial markers (vitamin $\mathrm{E}$ capsules) in place at a consistent series of points, slice thickness $=1.4 \mathrm{~mm}$ ) and procedures to measure their resting motor threshold, $5 \mathrm{~cm}$ anterior location, and targeted location and were then randomized prior to treatment. That is, the two sites were marked on the scalp (corresponding with the $5 \mathrm{~cm}$ and 


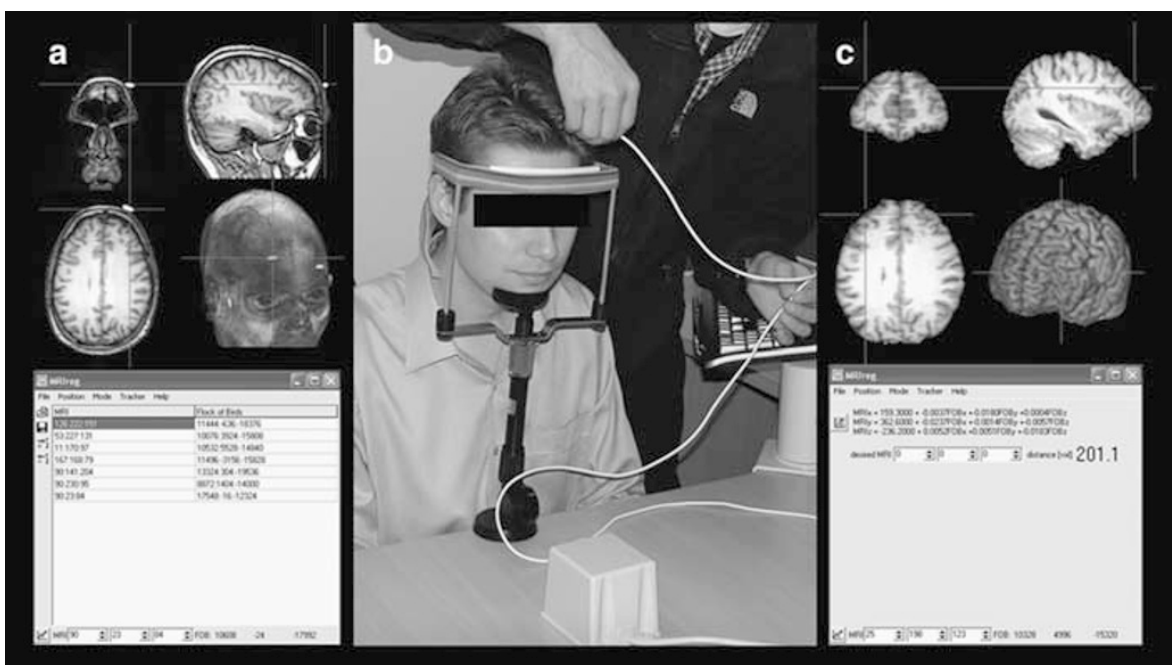

Figure I Illustration of the co-registration procedure with the miniBird system and MRIcro/MRIreg. (a) Registration of the subject's head to raw MRI scan. In this example, the fiducial marker $4 \mathrm{~cm}$ above the right eye is being registered. MRI coordinates are paired with coordinates in 3D space within the field of the magnetic field emitted by the device (small box in the foreground in (b)). (b) This shows the field emitter and the probe placed on the scalp. (c) This shows the Track mode in MRIreg: the cortical site displayed corresponds to the position in which the wand is placed on the scalp.

neuro-navigated location) with a marker during these measurement procedures, in all subjects, by one investigator. Then the randomization occurred. The mark corresponding to the group that the patient was not randomized to was then removed with an alcohol swab. The patient then received treatment by an investigator who had not participated in these procedures at the remaining site. The position of this site on the scalp was then used on all subsequent days. The position of this point was identified on subsequent days by recording and reusing measurements from the point to several anatomical regions including the pre-auricular point and the nasion.

The localization procedure used standard techniques for the Ascension miniBIRD (Ascension Technology Corporation, VT, USA) and MRIcro/MRIreg package to enable each participant's MR brain scan to be co-registered with their real head geometry (Rorden and Brett, 2000) (Figure 1):

(1) First, each scan was processed through SPM99, which reset the anterior commissure to 0,0,0 and warped each scan into Talairach space. The converted head image was scalp segmented using the brain extraction tool (Smith, 2002) to produce a brain-only image (ie without the skull and scalp). Both the brain and head images were rendered to produce two 3D images, one with anatomical surface markers intact (eg ears, fiducial markers) and one of the rendered cortical surface.

(2) The participant's head was placed in a frame. Each fiducial location on the MRI scan was then registered to the matching point on the person's head, by registering the location of those dots in $3 \mathrm{D}$ Talairach space with the MiniBIRD magnetic wand position on the head using MRIreg. MRIreg computes a multiple linear regression to map the marker coordinates onto MRI coordinates (least squares linear estimation).

(3) The reliability of the localization technique was confirmed by navigating the sensor/wand to known anatomical points such as nose or ears on the non-skull stripped scan.
(4) In the MRIreg 'tracker' mode, the second (skull-stripped cortical) image was used to identify the scalp surface position corresponding to the relevant cortical brain site identified as the target coil position. This was then marked on the scalp for subsequent use.

(5) To further increase our certainty in regard to the localization accuracy, the procedures were then repeated on the non-normalized scan to ensure the identified point corresponded to the appropriate position in the DLPFC based on gyral landmarks (junction of the middle and anterior thirds of the middle frontal gyrus).

\section{Site of Stimulation}

For stimulation of left DLPFC in the targeted group, we chose to aim for a spot between the center of BA 9 and the border of BA 9 and 46 based on the conservative definition of these areas produced by Rajkowska et al (Rajkowska and Goldman-Rakic, 1995). These are the cortical landmarks approximating the cytoarchitectural definitions of these brain regions. From Figure 13 (Rajkowska and GoldmanRakic, 1995), we selected the coordinates of $(y=45, z=35)$ and used an $x$-coordinate of -45 . Therefore, we aimed for the brain region identified by the coordinates $-45,45,35$. This localized stimulation approximately over the junction of the middle and anterior thirds of the middle frontal gyrus. This is also very close to the junction of 9 and 46 in the original map of Brodmann. The dorsal-ventral and anterior-posterior dimensions of both areas are $\leq 2 \mathrm{~cm}$ at this point (Rajkowska and Goldman-Rakic, 1995). Therefore, as the $70 \mathrm{~mm}$ figure-of- 8 coil produces stimulation over an area of up to $2 \mathrm{~cm}^{2}$ at a cortical depth of $2 \mathrm{~cm}$ (Jalinous, 1991), this should produce stimulation throughout parts of both areas 9 and 46 .

\section{Data Analysis}

We planned to compare clinical outcomes with repeated measures ANOVA models from baseline to week 4 (using 
the last observation carried forward method for all patients with at least one post-baseline evaluation). However, there was an imbalance in discontinuation rates, related apparently to an imbalance in clinical response rates, potentially biasing the model. Therefore, for the primary analysis, we conducted a mixed model analysis using the PROC MIXED procedure in SAS (SAS Version 9.1 SAS Institute Inc., Cary, NC, USA) with the fitted model incorporating fixed treatment and time effects and an interaction between treatment and time, and a REPEATED statement to indicate repeat measures. The PROC MIXED procedure uses a restricted maximum likelihood algorithm that enables specific modeling of the within-patient covariance structure. Using an AICC criterion to determine goodness of fit, an unstructured covariance structure was found to produce the best fit to the data. The Kenwood-Rogers method was used to estimate denominator degrees of freedom. The PROC MIXED procedure does not delete missing values list wise, but rather handles missing values by treating them as being missing at random. Empirical studies have confirmed the advantages of mixed models over last observation carried forward analysis, with mixed effects (Mallinckrodt et al, 2001a,b).

The $\chi^{2}$-squared tests were used to investigate the differences in the proportion of patients achieving response criteria ( $>50 \%$ reduction in MADRS score) between the groups and Fisher's exact test to analyze the differences in remission rates. Mixed models were also calculated for the cognitive test scores. All procedures were 2-tailed and significance was set at an $\alpha$ level of $<0.05$, with a Bonferroni correction used for post hoc comparisons. All statistical analysis was conducted with SPSS 14.0 (SPSS for Windows, 14.0, SPSS, Chicago, 2005) unless otherwise stated.

\section{RESULTS}

\section{Patient Groups}

A total of 51 patients, 22 males and 29 females, participated (age range 18-70, mean $41.3 \pm 12.4$ years) (Table 1). All the patients had a diagnosis of MDD (single episode $n=28$; MDD - relapse, $n=23$ ). Eleven patients met the criteria for comorbid social phobia, 18 generalized anxiety disorder, and 5 obsessive compulsive disorder. A total of 17 of 24 patients in the targeted group and 12 of the 27 in the standard group continued for the full 4 weeks of treatment $\left(\chi^{2}=3.6, p<0.06\right)$, the other patients ceasing after 3 weeks. The reasons for withdrawal included withdrawal of consent (one targeted, three standard group), unrelated medical illness (one standard group), family commitments (one standard group), and lack of response (six targeted group, ten standard group).

\section{Primary Outcomes}

When the MADRS outcomes were analyzed with the mixed model, there was a significant overall group effect $(p<0.05)$, a significant time effect $(p<0.0001)$, but a non-significant group $\times$ time interaction $(p>0.05)$ (Figure 2 , Table 2$)$. On planned post hoc testing, MADRS scores were significantly lower in the targeted group at 4 weeks $(p<0.05)$ but not 3 weeks $(p>0.05)$.
Ten patients $(42 \%)$ in the targeted group and five (18\%) in the standard group met response criteria $\left(\chi^{2}=3.3\right.$, $p<0.08)$. Seven $(30 \%)$ patients in the targeted group and three $(11 \%)$ in the standard group met remission criteria $(p>0.05)$.

\section{Secondary Outcomes}

There was a numerically greater improvement in the targeted group across all of the secondary outcome measures (Table 2). There were significant group effects on mixed model analysis for the Beck Depression Inventory, Brief Psychiatric Rating Scale, and GAF and a significant group $\times$ time interaction for the GAF.

\section{Cognition and Safety}

In regard to cognitive performance, there was an overall group improvement regarding word generation but not a between group difference (Table 3). There were no other significant changes.

There was no difference in the rate of side effects reported by the two groups. The patients reported site discomfort (four in the targeted and three in the standard group) and headache (most mild) (six in the targeted and six in the standard group). One patient in the targeted group reported nausea and a non-specific sense of disorientation. One patient in the standard group withdrew due to the development of shoulder-upper cervical back pain that was not felt to be related directly to the rTMS.

\section{DISCUSSION}

Our results suggest that the enhancement of rTMS localization with a structural MRI-based neuro-navigational procedure appears to have the capacity to improve response to the rTMS treatment in TRD. The primary result of the study was that there was a greater improvement in MADRS scores in the targeted group when compared with the standard treatment group, and this was also reflected in some of the secondary outcome variables, especially the GAF. However, the difference in the number of patients who progressed in the groups to the 4-week assessment does confound interpretation of the statistics, and suggests that these results require confirmation in future studies. Finally, there was no increase in side effects associated with targeted treatment.

There is growing and relatively consistent evidence that standard high-frequency left-sided rTMS treatment is more effective in the treatment of depression than sham stimulation (Fitzgerald, 2003). However, the degree of clinical response seen in most trials has been limited. Several approaches, including altering stimulation frequency (Fitzgerald et al, 2006b) and increasing dose (O'Reardon et al, 2007) have not markedly altered response rates. One previous randomized trial explored the use of a neuro-navigationally guided rTMS based on pretreatment positron emission tomography (PET) scans (Herwig et al, 2003) compared with sham. Active treatment was provided to an area of relative prefrontal cortical hypometabolism; but this was only detectable in 5 of the 13 patients. Other patients in the active group had stimulation provided to the 
Table I Demographic and Baseline Clinical Variables

\begin{tabular}{|c|c|c|c|c|}
\hline & \multicolumn{2}{|c|}{ Targeted group } & \multicolumn{2}{|c|}{ Control group } \\
\hline & Mean & SD & Mean & SD \\
\hline Age & 38.0 & 12.2 & 43.9 & 12.4 \\
\hline $\operatorname{Sex}(M / F)$ & $|3 /| \mid$ & & $9 / 18$ & \\
\hline \multicolumn{5}{|l|}{ Diagnosis } \\
\hline MDD_single episode & 10 & & 14 & \\
\hline MDD_relapse & 14 & & 13 & \\
\hline Number of failed antidepressant trials & 5.4 & 3.7 & 6.1 & 4.0 \\
\hline Number of depressive episodes & 4.2 & 5.1 & 4.1 & 4.1 \\
\hline MADRS & 30.8 & 4.5 & 33.4 & 7.3 \\
\hline $\mathrm{BDI}$ & 31.8 & 9.0 & 35.1 & 10.1 \\
\hline BPRS & 17.1 & 4.7 & 17.8 & 4.1 \\
\hline CORE & 4.2 & 4.5 & 4.3 & 4.8 \\
\hline CGI & 4.1 & 0.7 & 4.4 & 0.9 \\
\hline GAF & 49.3 & 6.4 & 47.7 & 7.7 \\
\hline $\begin{array}{l}\text { Concurrent antidepressant } \\
\text { medication } \\
\text { (none/SSRI/TCA/NAdrRI/SNRI/ } \\
\text { Combination) }\end{array}$ & $4 / 6 / 2 / 3 / 6 / 3$ & & $6 / 7 / 1 / 3 / 6 / 4$ & \\
\hline \multicolumn{5}{|l|}{ Comorbid/Diagnoses } \\
\hline Panic disorder & 2 & & 6 & \\
\hline OCD & 7 & & 7 & \\
\hline Social phobia & 10 & & 9 & \\
\hline GAD & 13 & & 13 & \\
\hline
\end{tabular}

SSRI, selective serotonin reuptake inhibitor; TCA, tricyclic antidepressant; NAdrRI, selective noradrenergic reuptake inhibitor; SNRI, serotonin and noradrenaline reuptake inhibitor; MDD, major depressive disorder; OCD, obsessive compulsive disorder; GAD, generalized anxiety disorder; MADRS, Montgomery-Asberg Depression Rating Scale; BDI, Beck Depression Inventory; CORE, CORE rating of Melancholia; CGI, Clinical Global Impression severity of illness rating; GAF, Global Assessment of Function.

left or right DLPFC with hemisphere allocated consecutively. The site of stimulation was a DLPFC region of interest defined as a target to the 'middle part of the middle frontal gyrus'. Active stimulation in the trial was better than sham, but there was no differential benefits for the patients for whom site was based on positron emission tomography scan compared with active treatment not based on positron emission tomography scan. Unfortunately, as the site within the DLPFC (as distinct from the hemisphere) did not vary, the study does not provide information about the overall value of the neuro-navigational localization of DLPFC.

A significant issue in our study was the site chosen for the targeted group. Although there is a relative consensus in the literature that the DLPFC is involved in the etiology of depression, the DLPFC is a relatively large area and there is no certainty as to which part of the DLPFC is likely to be involved critically in this disorder. In part to address this issue, we recently conducted a study, whereby we analyzed all functional neuroimaging studies that identified abnormalities in DLPFC in patients with depression (Fitzgerald

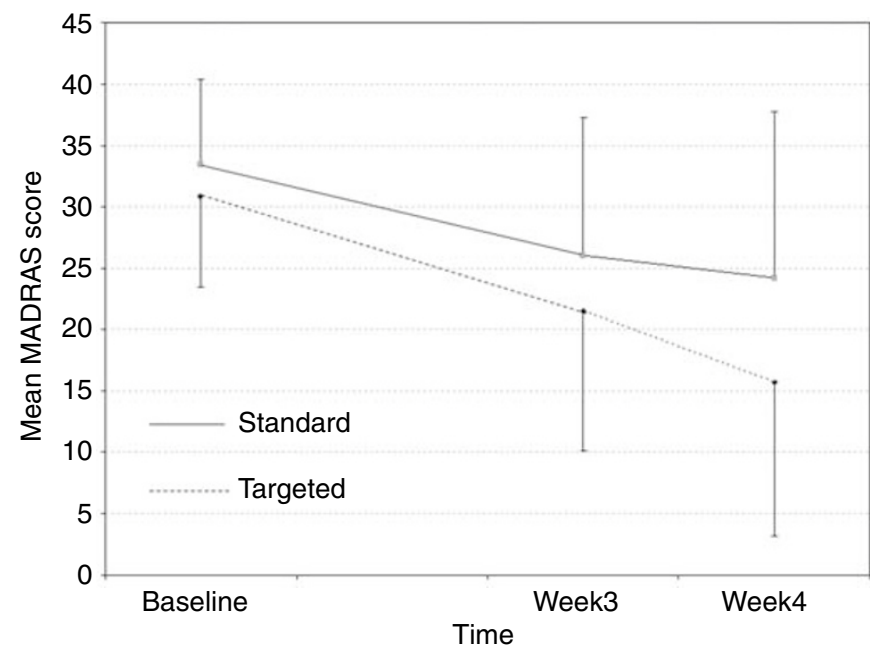

Figure 2 Change in Montgomery-Asberg Depression Rating Scale (MADRS) scores (least square means) over time.

et al, 2006c). Despite considerable heterogeneity in the results, there was some consistency in identification of changes in BA 9 across studies, although there was variability in the direction of the effects and hemisphere. Given this lack of consensus, for this study, we chose to be fairly conservative and target both BA 9 and 46 but with some bias towards the center of BA 9. The placement of the coil close to the boundary of these two areas, as defined by Rajkowska et al (Rajkowska and Goldman-Rakic, 1995), would ensure stimulation of the majority of both of these regions based on the conservative definition by these authors and the estimated $2 \mathrm{~cm}^{2}$ area covered by the standard $70 \mathrm{~mm}$ figure-of- 8 coil (Jalinous, 1991).

Unfortunately, as we did not record the anatomical site that was stimulated in the standard treatment group, we cannot be completely clear in regard to how much overlap there would have been between the targeted site of stimulation and the site of stimulation in the standard treatment group. However, the original study of the localization produced with standard $5 \mathrm{~cm}$ method conducted by Herwig et al (2001a) provides an estimation. The mean coordinates in that report on the $y$-and $z$-axis were 17 and $47(\mathrm{SD}=5,6)$ compared with the 45 and 35 we used. Therefore, given the variance in the measures by Herwig et al, it is likely that in most patients, we would have been stimulating approximately $2-3 \mathrm{~cm}$ anterior to the site that would have been selected using the $5 \mathrm{~cm}$ method. This would mean that there would be relatively limited overlap in the side of stimulation produced between the two sites given the size of the standard rTMS field. However, this form of across study comparison is limited by a number of potential biases, most importantly that a systematic error could be present because of the substantial variations between the study methods.

Another methodological issue worthy of consideration is that of the reliability of the neuro-navigational technique. The miniBIRD tracker system is described as having positional tracking accuracy of $1.8 \mathrm{~mm}$, although this is subject to potential degradation by surrounding magneticmetal objects (product documentation, Ascension TC, VT, USA). Translated to our application, this degree of accuracy 
Table 2 Treatment Response (Least Square Means for Each Outcome Measure)

\begin{tabular}{|c|c|c|c|c|c|c|c|c|c|}
\hline & \multicolumn{2}{|c|}{ Baseline } & \multicolumn{2}{|c|}{ Week 3} & \multicolumn{2}{|c|}{ Week 4} & \multicolumn{3}{|c|}{ Significance: F-statistic (df) } \\
\hline & Targeted & Standard & Targeted & Standard & Targeted & Standard & Time effect & Group Effect & $\begin{array}{l}\text { Group } \times \text { Time } \\
\text { interaction }\end{array}$ \\
\hline MADRS & $30.8 \pm 7.4$ & $33.4 \pm 6.9$ & $21.5 \pm 11.4$ & $26.2 \pm 11.2$ & $15.7 \pm 12.5$ & $24.2 \pm 13.5$ & $33.0(2,33)$ **** & $5.7(1,39)^{*}$ & $2.1(2,33)$ \\
\hline $\mathrm{BDI}$ & $31.8 \pm 12.0$ & $35.1 \pm 11.4$ & $16.1 \pm 15.1$ & $24.7 \pm 14.2$ & $10.8 \pm 14.2$ & $20.6 \pm 15.7$ & $38.4(2,39)^{* * * *}$ & $8.7(1,45)^{* * *}$ & I.3 $(2,39)$ \\
\hline BPRS & $17.1 \pm 5.4$ & $17.8 \pm 5 . \mid$ & $11.7 \pm 6.7$ & $13.6 \pm 6.3$ & $8.1 \pm 7.2$ & $12.6 \pm 7.4$ & $32.0(2,36)^{* * * *}$ & $3.8(1,42)^{\wedge}$ & $2.4(2,36)^{\wedge}$ \\
\hline CGl & $4.1 \pm 1.1$ & $4.4 \pm 1.0$ & $3.4 \pm 1.4$ & $4.0 \pm 1.3$ & $4.0 \pm 1.3$ & $3.6 \pm 1.5$ & $21.6(2,40)^{* * * *}$ & $4.5(1,46)^{*}$ & $1.6(2,40)$ \\
\hline GAF & $49.3 \pm 8.7$ & $47.7 \pm 7.9$ & $56.4 \pm 9.3$ & $53.0 \pm 8.8$ & $63.1 \pm 10.5$ & $54.7 \pm 9.1$ & $23.9(2,35)^{* * * *}$ & $3.3(1,46)^{\wedge}$ & $3.4(2,35)^{*}$ \\
\hline
\end{tabular}

* $p<0.05$, *** $p<0.01$, ****** $p<0.001$, ^ $p \leqslant 0.10$.

Mean Montgomery-Asberg Depression Rating Scale (MADRS), Beck Depression Inventory (BDI), Brief Psychiatric Rating Scale (BPRS), CORE melancholia rating scale, Clinical Global Improvement (CGI) and Global Assessment of Functioning (GAF) scores for each study visit.

Table 3 Cognitive Assessments (Least Square Means)

\begin{tabular}{|c|c|c|c|c|c|c|c|c|c|}
\hline & \multicolumn{2}{|c|}{ Baseline } & \multicolumn{2}{|c|}{ Week 3} & \multicolumn{2}{|c|}{ Trial End Point } & \multicolumn{3}{|c|}{ Significance: F-statistic (df) } \\
\hline & Targeted & Standard & Targeted & Standard & Targeted & Standard & Time effect & Group Effect & $\begin{array}{c}\text { Group } \times \text { Time } \\
\text { interaction }\end{array}$ \\
\hline BVMT TI & $5.3 \pm 2.9$ & $4.3 \pm 2.7$ & $6.5 \pm 3.3$ & $4.8 \pm 2.2$ & $6.8 \pm 3.2$ & $4.4 \pm 3.5$ & $4.2(2,34)$ & $6.9(1,46)$ & $1.6(2,34)$ \\
\hline BVMT T2 & $8.2 \pm 3.5$ & $6.5 \pm 3.3$ & $8.9 \pm 3.4$ & $6.8 \pm 3.3$ & $8.4 \pm 3.5$ & $5.8 \pm 3.5$ & $4.0(2,29)$ & $6.9(1,46)$ & $1.0(2,29)$ \\
\hline BVMT recognition. & $11.6 \pm 1.3$ & $11.4 \pm 1.3$ & $11.4 \pm 1.8$ & $11.2 \pm 1.8$ & $11.6 \pm 1.8$ & $11.5 \pm 1.3$ & $0.6(2,4 I)$ & $0.5(1,49)$ & $0.0(2,4 I)$ \\
\hline HVLT immediate recall & $26.1 \pm 4.0$ & $23.3 \pm 7.4$ & $24.6 \pm 6.8$ & $24.8 \pm 5.8$ & $27.0 \pm 4.6$ & $26.7 \pm 6.8$ & $2.2(2,35)$ & $0.7(1,45)$ & $1.2(2,35)$ \\
\hline HVLT delay & $9.8 \pm 2.8$ & $9.0 \pm 2.7$ & $8.8 \pm 3.3$ & $8.6 \pm 3.2$ & $8.7 \pm 4.3$ & $8.3 \pm 4.5$ & $3.6(2,36)$ & $0.4(1,45)$ & $0.4(2,36)$ \\
\hline HVLT discrimination index & $10.9 \pm 1.7$ & $10.9 \pm 1.6$ & $10.9 \pm 2.2$ & $10.8 \pm 2.2$ & $10.4 \pm 2.6$ & $9.9 \pm 2.8$ & $4.7(2,36)$ & $0.2(1,45)$ & $0.5(2,36)$ \\
\hline COWAT & $38.8 \pm 13.5$ & $35.3 \pm 4.7$ & $44.7 \pm \mid 4.1$ & $38.0 \pm 13.7$ & $46.1 \pm 13.5$ & $39.7 \pm 18.1$ & $8.0(2,31)^{*}$ & $2.8(1,47)$ & I.2 (2,3। $)$ \\
\hline
\end{tabular}

BVMT, Brief Visuospatial Memory Test-Revised (Time I, 2 and 3=TI-3); HVLT, Hopkins Verbal Learning Test; COWAT, Controlled Oral Word Association Test. $* p<0.01$

is highly acceptable as it is well within the geographical size of the regions we are trying to locate. More critically, it is of considerable importance to ensure that the co-registration procedure is conducted in such a way to ensure reliable registration of the brain to MRI space. In each individual subject, we tried to ensure that this was the case by testing the co-registration with the software in 'tracker' mode. By placing the tracker over known anatomical landmarks, it was clear whether or not the same regions were identified accurately on the non-skull stripped MRI scan. For approximately $30 \%$ of the occasions, this was sufficiently inaccurate to ensure that we repeated the co-registration procedure or added several more registration points to achieve a better response. In all cases, this resulted in a satisfactory degree of co-registration. However, although we were able to confidently achieve what appeared to be a successful co-registration for each subject, we have not obtained repeat-reliability data within subjects across time.

It is useful to consider whether the technique of this sort has practical applicability. The miniBIRD is quite inexpensive and the MRIreg and MRIcro software available as freeware. These are also relatively easy to use with training. The image processing and software requirements to normalize the scans to allow localization based on Talairach coordinates as well as anatomical landmarks is more complicated, but it is likely that a similar effect could be purely achieved with localization based on neuro-anatomical landmarks. If a large-scale study was to be designed to test the practical implementation of a targeting technique, this would seem to be a very reasonable compromise. However, there may be another alternative to conducting the full targeting procedure. This may be as simple as adjusting the measurement process to ensure a more accurate and reliable targeting of true DLPFC. Our results would suggest that measurement at least $7 \mathrm{~cm}$ anterior to the site of optimal stimulation of the abductor pollicis brevis muscle may improve rTMS response rates, perhaps with a correction for head size. There is also support for a more lateral coil location from recent data (Hasey et al, 2008). This is an important and practical consideration, as adopting a slightly altered measurement would be considerably simpler and more reliably applicable than using a 
neuro-navigated approach. Unfortunately, to determine this will require further study: into the degree of intra-individual variability in anatomical relatedness between motor and prefrontal cortex, and perhaps a similar trial to the one presented here comparing neuro-navigation to stimulation at a more anterior-fixed location.

It is important to note that the main findings of this study are limited by a difference in dropout rates experienced by the two groups between weeks 3 and 4 and the group $\times$ time interaction for the MADRS did not reach significance for the mixed model analysis. Given the structure of the trial, it is likely that the greater dropout rate in the standard group was related to poorer overall response in these patients (especially patients not meeting response criteria at 3 weeks). This is reflected in the larger proportion of subjects in the non-response category for dropout in the standard group. Although three dropouts in the standard group were recorded as 'withdrawal of consent', it seems likely that this was also influenced by lack of clinical improvement. Adding these two categories indicated a dropout rate of $7 / 24$ for targeted and 13/27 for the standard group. Although the differences in the dropout rate were not significant $(p<0.06)$, there is certainly a strong indication that targeted treatment was associated with a reduced dropout because of the lack of efficacy supporting the notion that this intervention was more effective. It is also noted that this imbalance in dropout rates would actually bias towards a smaller, not larger, between-group difference at 4 weeks. Therefore, although the difference in dropouts does affect our capacity to interpret the 4-week results in the mixed model analysis, most likely this bias will not contribute to a false-positive result. The positive effect of the intervention is also supported by the significant group by time interaction on the GAF. The marginal effects are likely to at least, in part, reflect inherent difficulties establishing statistically significant differences between the two active treatments.

There are some other limitations of the clinical trial worthy of mention. First, the results may have been confounded by non-specific effects given that we have not included a sham control group. Second, whilst we have provided treatment for 4 weeks, studies are now testing rTMS methods over longer periods of time (Fitzgerald et al, 2006a; O'Reardon et al, 2007). It is possible that the standard group could 'catch up' in their response rates with a longer duration of treatment, although there is no clear rationale as to why targeting would accelerate rather than enhance responses. However, further methodological refinement could result in greater treatment effects. For example, the navigational technique, we used, was offline and a greater degree of effectiveness may have been achieved in this group, had we used a technique to continually monitor the coil position during treatment. Targeting treatment based on individual functional neuroimaging may also result in enhanced targeting of either a specific brain region or, alternatively, area of abnormal activation. In addition, it is important to note that although the mixed model procedure used is superior to using a last observation carried forward analysis, in which there is an imbalanced dropout rate, the results need to be interpreted with caution given that no models work particularly well under these circumstances. Finally, the patients in our study had a high rate of anxiety disorders. Although mood and anxiety disorders are frequently comorbid, it is possible that the response rates to rTMS in general may be different in a group of patients with a single disorder, perhaps of the melancholic depression type (Fitzgerald et al, 2003).

In conclusion, the results of this study suggest that more accurate targeting of DLPFC, specifically BA 9 and the dorsal portion of $\mathrm{BA} 46$, may be more effective than standard rTMS localization methods. However, this effect does require replication in larger samples. It was at no sideeffect cost and the method was implemented with relatively limited technical complexity. Research is also required to establish whether similar benefits may be achieved with simpler methods of reliably stimulating the target site, eg based on EEG coordinates. It would also be of interest to establish whether individual structural and functional connectivity maps of DLPFC could be used to identify the most ideal target for stimulation.

\section{ACKNOWLEDGEMENTS}

PBF was supported by a Practitioner Fellowship grant from National Health and Medical Research Council (NHMRC) and PBF and ZJD by NARSAD Young Investigator awards. This study was supported in part by a NHMRC project Grant (436710) and the Neurosciences Australia Clinical Neurobiology of Psychiatry Platform. We would like to thank the patients whose participation was essential in the successful completion of this study. The Clinicaltrials. gov trial registration number is NCT00168376 (http:// clinicaltrials.gov/ct/show/NCT00168376?order =1).

\section{DISCLOSURE}

PBF and ZJD have received support for research conducted with Neuronetics Inc, a TMS equipment manufacturer and have no other relevant biomedical financial interests. $\mathrm{KH}$, SMQ, JJM, SH, RS, GB, MB, and JK have no relevant biomedical financial interests or conflicts to declare.

\section{REFERENCES}

Arnold SE, Franz BR, Gur RC, Gur RE, Shapiro RM, Moberg PJ et al (1995). Smaller neuron size in schizophrenia in hippocampal subfields that mediate cortical-hippocampal interactions. Am J Psychiatry 152: 738-748.

American Psychiatric Association (1994). DSM-IV. American Psychiatric Association Press: Washington, DC.

Beck A, Ward C, Mendelson M, Mock J, Erbaugh J (1961). An inventory for measuring depression. Arch Gen Psychiatry 4: 561-571.

Benedict RH, Schretlen D, Groninger L, Dobraski M, Shpritz B (1996). Revision of the brief visuospatial memory test: studies of normal performance, reliability, and validity. Psychol Assess 8: $145-153$.

Benton AL, Hansher K (1996). Multilingual Aphasia Examination, AJA Associations 1989, Iowa City, USA.

Fava M (2003). Diagnosis and definition of treatment-resistant depression. Biol Psychiatry 53: 649-659.

Fitzgerald P (2003). Is it time to introduce repetitive transcranial magnetic stimulation into standard clinical practice for the treatment of depressive disorders? Aust NZ J Psychiatry 37: 5-11; discussion 12-14. 
Fitzgerald PB (2006). A review of development in brain stimulation in the treatment of psychiatric disorders. Curr Psychiatry Rev 2: 199-205.

Fitzgerald PB, Benitez J, de Castella A, Daskalakis ZJ, Brown TL, Kulkarni J (2006a). A randomized, controlled trial of sequential bilateral repetitive transcranial magnetic stimulation for treatment-resistant depression. Am J Psychiatry 163: 88-94.

Fitzgerald PB, Brown TL, Marston NA, Daskalakis ZJ, De Castella A, Kulkarni J (2003). Transcranial magnetic stimulation in the treatment of depression: a double-blind, placebo-controlled trial. Arch Gen Psychiatry 60: 1002-1008.

Fitzgerald PB, Huntsman S, Gunewardene R, Kulkarni J, Daskalakis ZJ (2006b). A randomized trial of low-frequency rightprefrontal-cortex transcranial magnetic stimulation as augmentation in treatment-resistant major depression. Int J Neuropsychopharmacol 9: 655-666.

Fitzgerald PB, Oxley TJ, Laird AR, Kulkarni J, Egan GF, Daskalakis ZJ (2006c). An analysis of functional neuroimaging studies of dorsolateral prefrontal cortical activity in depression. Psychiatry Res 148: 33-45.

George MS, Nahas Z, Molloy M, Speer AM, Oliver NC, Li XB et al (2000). A controlled trial of daily left prefrontal cortex TMS for treating depression. Biol Psychiatry 48: 962-970.

George MS, Wassermann EM, Williams WA, Callahan A, Ketter TA, Basser P et al (1995). Daily repetitive transcranial magnetic stimulation (rTMS) improves mood in depression. Neuroreport 6: $1853-1856$.

Guy W (1976). Clinical Global Impressions. ECDEU Assessment manual for psychopharmacology revised. National Institute of Mental Health: Rockville, MD, 218-222.

Hasey D, Shew MHC, Heaslip J, MacCrimmon D, Abraham G, Milev R et al (2008). The antidepressant effect of transcranial magnetic stimulation is strongly correlated with the distance of the coil in the midline. Biol Psychiatry 63: 295S.

Herwig U, Lampe Y, Juengling FD, Wunderlich A, Walter $\mathrm{H}$, Spitzer $M$ et al (2003). Add-on rTMS for treatment of depression: a pilot study using stereotaxic coil-navigation according to PET data. J Psychiatr Res 37: 267-275.

Herwig U, Padberg F, Unger J, Spitzer M, Schonfeldt-Lecuona C (2001a). Transcranial magnetic stimulation in therapy studies: examination of the reliability of "standard" coil positioning by neuronavigation. Biol Psychiatry 50: 58-61.

Herwig U, Schonfeldt-Lecuona C, Wunderlich AP, von Tiesenhausen $\mathrm{C}$, Thielscher A, Walter $\mathrm{H}$ et al (2001b). The navigation of transcranial magnetic stimulation. Psychiatry Res 108: 123-131.

Hickie I, Parsonage B, Parker G (1990). Prediction of response to electroconvulsive therapy: preliminary validation of a sign-based typology of depression. B J Psychiatry 157: 65-71.

Jalinous R (1991). Technical and practical aspects of magnetic nerve stimulation. J Clin Neurophysiol 8: 10-25.
Krings T, Chiappa KH, Foltys H, Reinges MH, Cosgrove GR, Thron A (2001). Introducing navigated transcranial magnetic stimulation as a refined brain mapping methodology. Neurosurg Rev 24: 171-179.

Loo CK, Mitchell PB (2005). A review of the efficacy of transcranial magnetic stimulation (TMS) treatment for depression, and current and future strategies to optimize efficacy. J Affect Disord 88: $255-267$.

Mallinckrodt C, Clark W, David S (2001a). Accounting for dropout bias using mixed-effects models. J Biopharm Stat 11: 9-21.

Mallinckrodt C, Clark W, David S (2001b). Type I error rates from mixed effects model repeated measures compared with fixed effects ANOVA with missing values imputed via LOCF. Drug Inf J 35: 1215-1225.

Montgomery SA, Asberg M (1979). A new depression scale designed to be sensitive to change. Br J Psychiatry 134: 382-389.

O'Reardon J, Solvason B, Janicak P, Sampson S, Isenberg K, Nahas Z et al (2007). Efficacy and safety of transcranial magnetic stimulation in the acute treatment of major depression: a multisite randomized controlled trial. Biol Psychiatry 62: 1208-1216.

Overall JE, Gorham D (1962). The brief psychiatric rating scale. Psychol Rep 10: 799-812.

Pascual-Leone A, Rubio B, Pallardo F, Catala MD (1996). Rapid-rate transcranial magnetic stimulation of the left dorsolateral prefrontal cortex in drug-resistant depression. Lancet 348: 233-237.

Pridmore S, Fernandes Filho JA, Nahas Z, Liberatos C, George MS (1998). Motor threshold in transcranial magnetic stimulation: a comparison of a neurophysiological method and a visualization of movement method. J ECT 14: 25-27.

Rajkowska G, Goldman-Rakic PS (1995). Cytoarchitectonic definition of prefrontal areas in the normal human cortex: II. Variability in locations of areas 9 and 46 and relationship to the Talairach Coordinate System. Cereb Cortex 5: 323-337.

Rorden C, Brett M (2000). Stereotaxic display of brain lesions. Behav Neurol 12: 191-200.

Schonfeldt-Lecuona C, Thielscher A, Freudenmann RW, Kron M, Spitzer M, Herwig U (2005). Accuracy of stereotaxic positioning of transcranial magnetic stimulation. Brain Topogr 17: 253-259.

Sheehan DV, Lecrubier Y, Sheehan KH, Amorim P, Janavs J, Weiller E et al (1998). The Mini-International Neuropsychiatric Interview (M.I.N.I.): the development and validation of a structured diagnostic psychiatric interview for DSM-IV and ICD-10. J Clin Psychiatry 59(Suppl 20): 22-33.

Smith SM (2002). Fast robust automated brain extraction. Hum Brain Mapp 17: 143-155.

Thase ME, Rush AJ (1997). When at first you don't succeed: sequential strategies for antidepressant nonresponders. J Clin Psychiatry 58(Suppl 13): 23-29.

Wechsler D (1997). Wechsler Adult Intelligence Scale. Harcourt Brace \& Company: San Antonio. 